\title{
Pancreatic neuroendocrine tumor with ectopic adrenocorticotropic hormone syndrome: a case report and 5-year follow-up
}

\author{
Mao Zheng ${ }^{1), 2)}$, Li Chen ${ }^{3)}$, Xiaomin $\mathrm{Nie}^{1), 2)}$, Dong Wang ${ }^{1), 2)}$, Jie Zhu ${ }^{1), 2)}$, Wei Wang ${ }^{1), 2)}$, An $\operatorname{Ren}^{1)}$ and \\ Shandong $\mathrm{Ye}^{1), 2)}$ \\ 1) Department of Endocrinology, The First Affiliated Hospital of USTC, Division of Life Sciences and Medicine, University of Science \\ and Technology of China, Hefei, Anhui Province, 230001, China \\ 2) Laboratory of Diabetes, Department of Endocrinology, The First Affiliated Hospital of USTC, Hefei, Anhui Province, 230001, \\ China \\ 3) Department of Endocrinology, The Third Affiliated Hospital of Anhui Medical University (The First People's Hospital of Hefei), \\ Hefei, Anhui Province, 230601, China
}

\begin{abstract}
Pancreatic neuroendocrine tumors (P-NETs) secreting ectopic adrenocorticotropic hormones (ACTH) are rare and often delayed in diagnosis due to their atypical clinical characteristics. Here, we describe a case of P-NET in the pancreatic tail. The tumor had metastasized to the liver and secreted gastrin and ACTH. A 60-year-old female patient was diagnosed with gastrinoma in the pancreatic tail with liver metastases in 2015. After 3 months, the patient presented refractory hypokalemia and thyroid dysfunction. The final diagnosis was P-NET with ectopic ACTH syndrome (EAS). After cytoreductive surgery and the use of long-acting somatostatin analogs, plasma potassium levels and thyroid function were effectively corrected. Although Sandostatin LAR ${ }^{\circledR}$ Depot and proton pump inhibitors (PPIs) were used throughout the follow-up period, the tumor relapsed 4 years later. After aggressive treatment, including right hepatectomy, microwave coagulation of the left liver, and cholecystectomy, the tumor returned 4 months later. Finally, the patient underwent three hepatic artery embolizations and 12 courses of CAPTEM regimen chemotherapy. The markers of disease were almost maintained in the normal ranges until now. We have followed up on this case for more than 5 years. A timely and comprehensive examination of hormones and immunohistochemistry is essential. The prognosis of P-NET is poor. Regular long-term follow-up and the application of combined therapies are helpful to control the disease and improve the prognosis.
\end{abstract}

Key words: Pancreatic neuroendocrine tumor, Gastrin, Ectopic adrenocorticotropic hormone syndrome, Pancreas, Liver metastasis

PANCREATIC NEUROENDOCRINE TUMORS (PNETs) are a group of heterogeneous tumors derived from pluripotent stem cells of the neuroendocrine system. P-NETs are rare and account for $1-2 \%$ of all pancreatic neoplasms [1]. Neuroendocrine tumors and metastatic lesions can secrete multiple hormones, including insulin, gastrin, adrenocorticotropic hormone $(\mathrm{ACTH})$,

Submitted May 14, 2021; Accepted Sep. 6, 2021 as EJ21-0297 Released online in J-STAGE as advance publication Oct. 9, 2021 Correspondence to: Shandong Ye, Department of Endocrinology, The First Affiliated Hospital of USTC, Division of Life Sciences and Medicine, University of Science and Technology of China. No. 17, Lujiang Road, Hefei, Anhui Province 230001, P. R. China.

E-mail: ysd196406@163.com

Correspondence to: Li Chen, Department of Endocrinology, The Third Affiliated Hospital of Anhui Medical University (The First People's Hospital of Hefei). No. 3200, Changsha Road, Hefei, Anhui Province 230601, P. R. China.

E-mail: 294598984@qq.com corticotropin-releasing hormone, growth hormone, parathyroid hormone (PTH) and calcitonin [2, 3]. Insulinoma and gastrinoma are the most common types of P-NETs, while pancreatic gastrinoma with ectopic ACTH syndrome (EAS) is rare and tends to be misdiagnosed. Here, we report a case of P-NET in the pancreatic tail. The tumor had metastasized to the liver and secreted gastrin and ACTH. We followed up with the patient for more than 5 years.

\section{Case Report}

A 60-year-old woman came to the gastroenterology clinic of our hospital in August 2015. She had symptoms of recurring acid reflux and burning pain in the upper abdomen for nearly 6 years. The symptoms could not be alleviated completely by oral proton pump inhibitors (PPIs). She also complained of nausea, vomiting and 
diarrhea for the last 2 months. Multiple duodenal ulcers and proctitis were found by gastroscopy and colonoscopy. After that, abdominal enhanced computerized tomography (CT) was conducted, which revealed a mass located in the pancreatic tail and multiple metastasized tumors located in the liver. Laboratory tests revealed a significant increase in gastrin 17. The pathological report of the liver biopsy revealed G2 NETs. The patient was finally diagnosed with pancreatic gastrinoma with liver metastasis. Rather than surgery, she chose the combination treatment of Sandostatin LAR ${ }^{\circledR}$ Depot (octreotide acetate for injectable suspension microspheres at $20 \mathrm{mg}$ every $28 \mathrm{~d}$ ) and PPI (nesomeprazole at $40 \mathrm{mg}$ every day). Fortunately, acid reflux and diarrhea were relieved, and the regimen was sustained for 3 months. In November 2015, the patient returned to the outpatient clinic for persistent weakness in both lower extremities and increased nocturia and facial edema for 2 weeks. Laboratory tests showed hypokalemia and thyroid dysfunction (Table 1), and then the patient was referred to an endocrinologist to seek the etiology of hypokalemia. She denied vomiting, having diarrhea and practicing

Table 1 General lab data*

\begin{tabular}{lcc}
\hline Parameters & Results & Reference values \\
\hline Serum potassium $(\mathrm{mmol} / \mathrm{L})$ & 2.45 & $(3.5-5.3)$ \\
\hline Fasting glucose $(\mathrm{mmol} / \mathrm{L})$ & 6.3 & $(3.9-6.1)$ \\
HbAlc $(\%)$ & 6.7 & $(4-6)$ \\
Gastrin-17 $(\mathrm{pmol} / \mathrm{L})$ & 13 & $(1-7.0)$ \\
Thyrotropin $(\mathrm{mIU} / \mathrm{L})$ & 0.28 & $(0.27-4.2)$ \\
Free triiodothyroninen $(\mathrm{pmol} / \mathrm{L})$ & $<1.0$ & $(3.1-6.8)$ \\
Free thyroxine $(\mathrm{pmol} / \mathrm{L})$ & 5.68 & $(12-22)$ \\
\hline
\end{tabular}

* All tests were performed in November 2015 when the patient was admitted to the Endocrinology Department. anorexia recently. Hypokalemia was stubborn and could not be easily corrected by potassium supplementation. Physical examination showed mild moon-face and a slight mustache on the upper lip. The levels of cortisol were dramatically high, and the cortisol circadian rhythm disappeared. Low-dose and high-dose dexamethasone suppression tests failed to suppress the levels of cortisol (Table 2). Bone mineral density detected by the dual $\mathrm{X}$-ray method and magnetic resonance imaging (MRI) suggested lumbar spine osteoporosis with compression fracture of the third lumbar vertebra. Abdominal CT revealed a $15-\mathrm{mm}$ mass located in the pancreatic tail and multiple masses located in the liver, which were considered metastatic cancer. The bilateral adrenal glands were also diffusely thickened (Fig. 1). Pituitary MR showed partial vacuolar sella. Immunohistochemistry (IHC) staining of the liver biopsy samples from August 2015 was tested for ACTH, which was positive (Fig. S1). Therefore, we concluded that the patient had a P-NET accompanied by liver metastasis and EAS. Examinations including serum calcium, parathormone and parathyroid gland ultrasound were also performed. Pituitary tumor, hyperparathyroidism and adrenal adenoma were eventually excluded. No evidence of multiple endocrine neoplasia 1 was found.

In Jan 2016, an open surgery plan including distal pancreatectomy, total splenectomy, partial liver tumors resection and microwave coagulation cytoreduction was performed to minimize the tumor burden. Pathological findings suggested P-NEN, G2, with liver metastases. IHC staining of tumors from the pancreatic tail and liver showed positivity for ACTH and gastrin (Fig. S2). Serum potassium and thyroid function returned to normal ranges soon after the surgery. Two months after the surgery, the levels of cortisol and ACTH gradually declined to the normal range (Fig. 2). Sandostatin LAR ${ }^{\circledR}$ Depot and PPIs were used throughout the follow-up.

Table 2 Cortisol circadian rhythm and DXM suppression tests*

\begin{tabular}{lrrrc}
\hline & Time point & $\begin{array}{c}\text { Serum cortisol } \\
(\mathrm{nmol} / \mathrm{L})\end{array}$ & $\begin{array}{c}\text { Plasma ACTH } \\
(\mathrm{pg} / \mathrm{mL})\end{array}$ & $\begin{array}{c}\text { Urinary free } \\
\text { cortisol excretion } \\
(\mathrm{nmol} / \mathrm{D})^{\#}\end{array}$ \\
\hline Baseline & $8: 00$ & 1,101 & 311 & 18,656 \\
& $16: 00$ & 985 & 323 & \\
Low-dose dexamethasone & $24: 00$ & 910 & 267 & 18,630 \\
suppression test & 1,164 & 315 & 16,000 \\
$\begin{array}{l}\text { High-dose dexamethasone } \\
\text { suppression test }\end{array}$ & 1,222 & 275 & \\
\hline * Performed in Nov 2015 & & & \\
\# Samples were diluted 10-fold by saline solution & & & \\
\end{tabular}



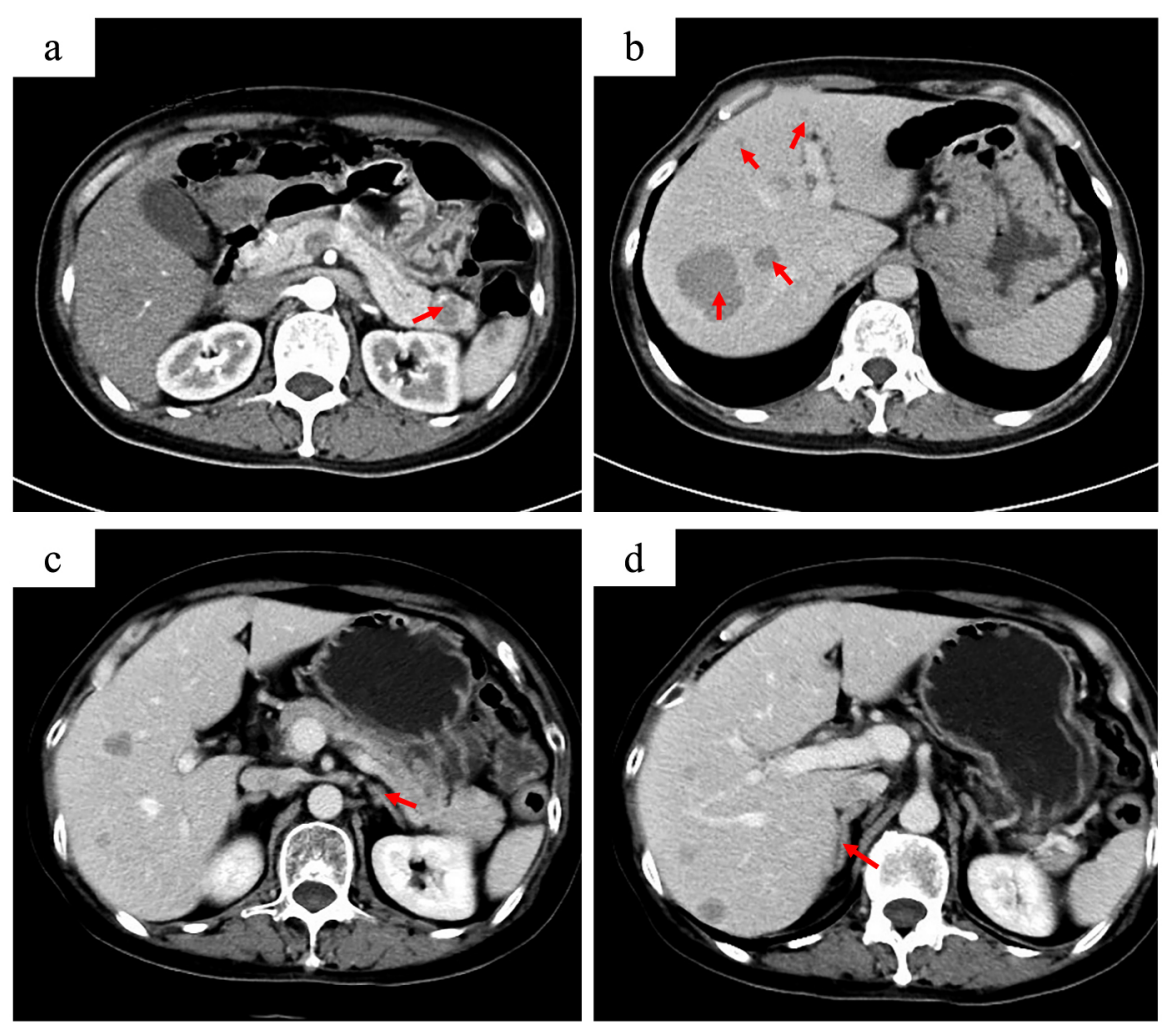

Fig. 1 Enhanced abdominal CT taken in November 2015

a. Gastrinoma of the pancreatic tail. b. Liver metastasis. c. Diffusely thickened left adrenal gland. d. Diffusely thickened right internal adrenal limb.

Serum levels of potassium, cortisol and ACTH were monitored regularly and maintained normal since then.

In Jan 2020, the symptoms of lower limb fatigue and facial edema, which had been absent for 4 years after the first surgery, returned, as did hypokalemia, low serum $\mathrm{TSH}$, high serum cortisol and gastrin 17. Enhanced abdominal MR revealed multiple metastases within the liver (Fig. 3). Then, right hepatectomy, microwave coagulation of the left liver and cholecystectomy were performed. The pathological report indicated a welldifferentiated NET (G2 stage) (Fig. 4). Serum potassium, thyroid function, cortisol, and ACTH temporarily returned to normal ranges after surgery. However, the tumor relapsed only 4 months later (Fig. 2). PET-CT showed that multiple metastases appeared again in the residual liver tissue with strong and positive somatostatin receptor imaging (Fig. 5). Finally, from January 2020 to January 2021, the patient underwent three hepatic artery embolizations and 12 courses of capecitabine plus temozolomide (CAPTEM regimen) chemotherapy (Fig. 6). Serum levels of potassium, gastrinoma, cortisol, and ACTH were evaluated every 1-2 months, and they were maintained in the normal ranges until now (Fig. 2).

\section{Discussion}

Patients with P-NETs often first visited the Department of Gastroenterology for digestive tract symptoms. Liver metastasis is often present on the most involved organ, which is a sign of poor prognosis [4]. The incidence of P-NETs with EAS is less than $1 / 1,000,000[5,6]$ and they are often delayed in diagnosis due to their atypical clinical characteristics. In this case, the patient received a P-NET secreting gastrin and ACTH simultaneously. In the early stage, she was first diagnosed with gastrinoma with liver metastasis. Only serum gastrin was proved to be in a high level. Serum insulin, glucagon and other hormones were not detected at the first visit of the patient. Then, mild cushingoid features and intractable hypokalemia occurred 3 months after the diagnosis. More comprehensive consideration was needed in our further clinical diagnosis and treatment. EAS was finally confirmed by elevated serum levels of cortisol and ACTH, positive results of the high-dose dexamethasone suppression test, and positive immunostaining for ACTH in liver biopsy specimens. Postoperative IHC also confirmed that gastrin and ACTH were positive in pancreatic tail tumors and liver metastases. 

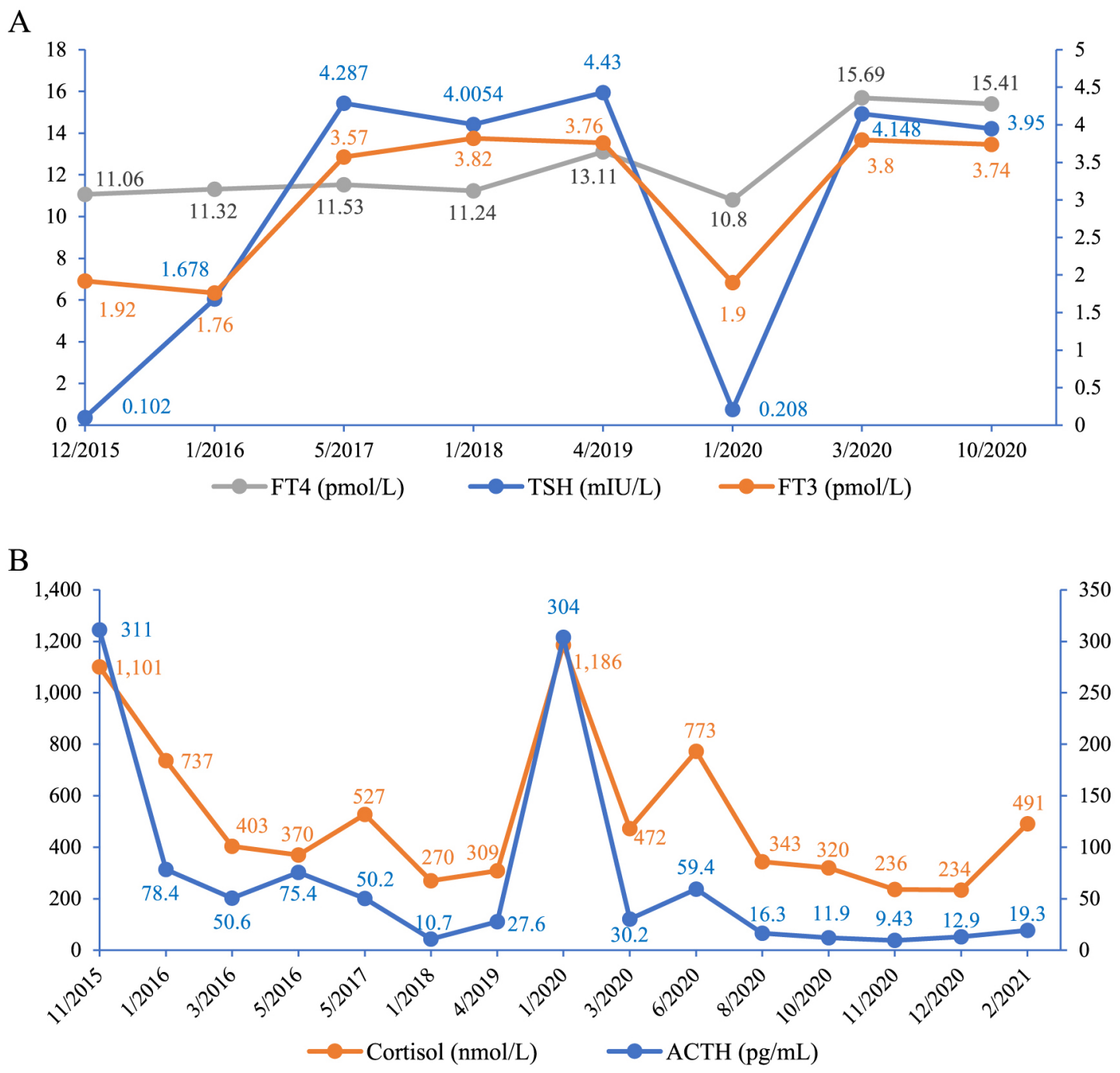

Fig. 2 Thyroid hormone level and TSH level in correlation with fluctuation in ACTH and cortisol levels during disease. Fig. 2A showed the thyroid function; Fig. 2B showed the adrenocortical function.

We speculated on the possible causes for the delayed manifestation of Cushing syndrome (CS). First, the manifestation may be subclinical at the early stage of the disease, which tends to be easily ignored and misdiagnosed. The patient merely showed atypical cushingoid features, impaired glucose tolerance and osteoporosis with nonviolent fractures in the early stage. Second, PNETs might initially secrete a small amount of ACTH. With the enlargement and metastasis of the tumor, the clinical symptoms of CS gradually occurred, accompanied by excessive secretion of ACTH. Maragliano R et al. [7] reported that in $71 \%$ of P-NETs that secreted ACTH and gastrin, the symptoms of EAS appeared 33 months later than those of gastrinoma on average.

The pathophysiology of hypothyroidism may have several causes. First, chronic hypercortisolism plays an important role in the change of thyroid function: (1) At the hypothalamic level, chronic hypercortisolism can reduce thyrotropin-releasing hormone (TRH) expression and increase somatostatin release, which in turn inhibits TSH release from thyrotroph cells [8]. In addition, the glucocorticoid receptor (GR) has been demonstrated to exist in TRH neurons in the paraventricular nucleus of the hypothalamus $[9,10]$. Inappropriate cortisol secretion was related to GR downregulation [11], which can also lead to the downregulation of TRH $[12,13]$. (2) At the pituitary level, hypercortisolism directly reduces TSH secretion and TSH pulse amplitude [8, 14]. It can also activate somatostatin receptor (SSTR) 2 and SSTR5 on TSH neurons and inhibit the release of TSH [15]. (3) Hypercortisolism also inhibits peripheral conversion from T4 to T3 by inhibiting 5'-deiodase activity, leading to nonthyroidal illness syndrome [16-19]. (4) A glucocorticoid-induced decrease in thyroid hormonebinding globulin (TBG) resulted in decreased $\mathrm{T} 3$ and $\mathrm{T} 4$ [20-22]. Second, the vacuolar sella may affect the 

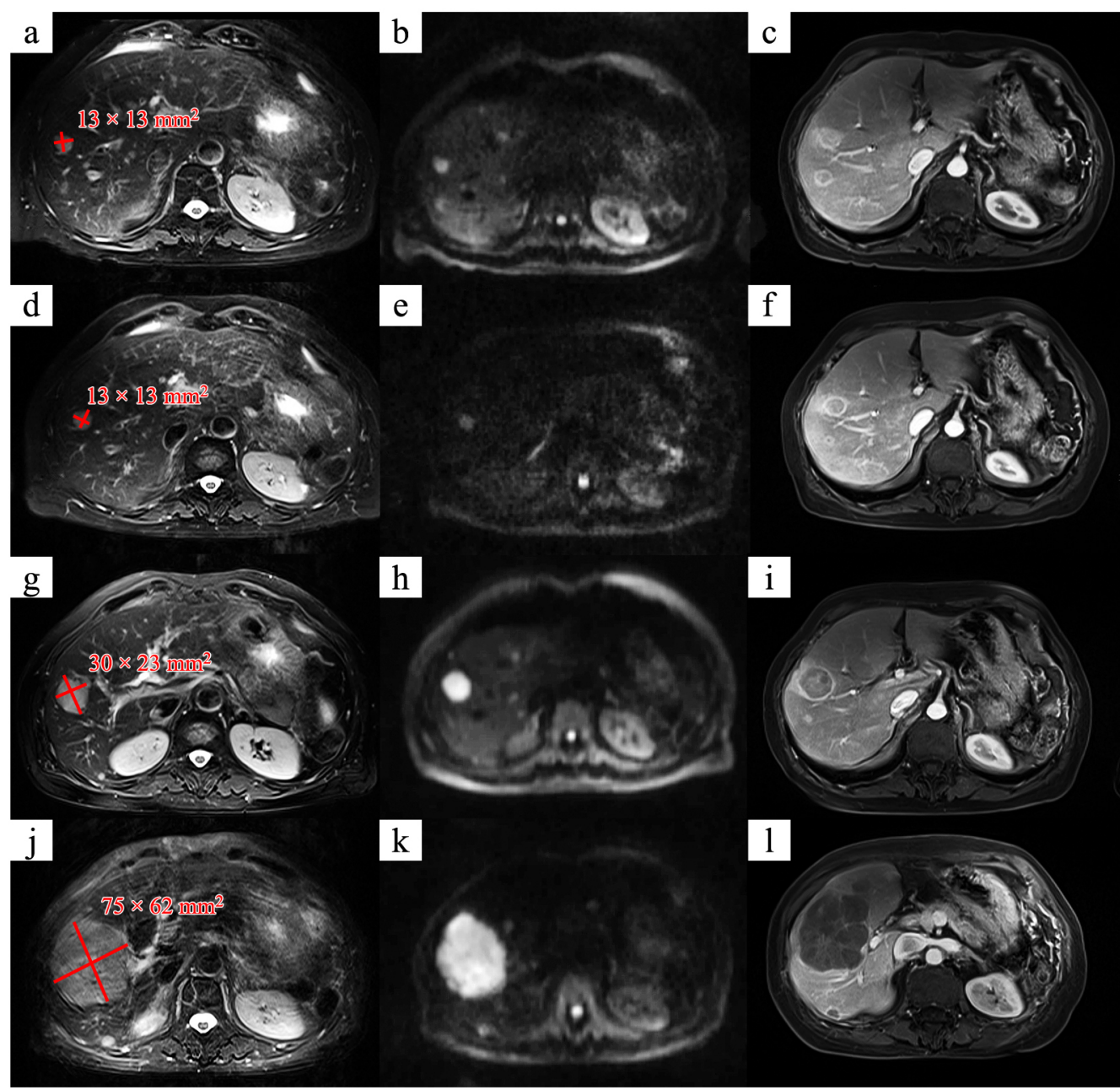

$\mathrm{k}$
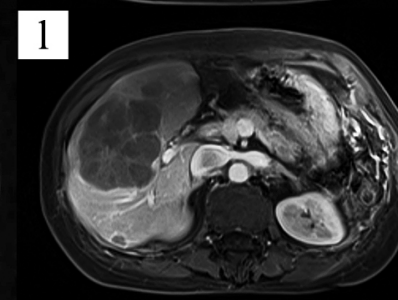

Fig. 3 Abdominal enhanced MR in September 2016 (a, b, c), July 2017 (d, e, f), November 2018 (g, h, i) and January 2020 (j, k, l)

T2WI fat-inhibiting sequence (T2WI-FS), diffusion-weighted sequence (DWI), and enhanced sequence were listed in three columns, respectively. The images in September 2016 showed multiple lesions in the right lobe of the liver. A slightly high signal in T2WI-FS and a high signal in DWI, and an enhancement were observed in these lesions. The liver lesions did not change substantially in the images in September 2017 (shown by the red markers). In the images of November 2018, a lesion in the hepatic segment 6 was significantly enlarged (shown by the red markers), with a high DWI signal and a ring enhancement. Then, in January 2020, this lesion was further enlarged into the sizes of $75 \mathrm{~mm} \times 62 \mathrm{~mm}$, with a high DWI signal and a slight enhancement.

function of the anterior pituitary lobe and cause central hypothyroidism [23, 24]. Third, long-term injection of somatostatin analogs could suppress serum TSH through direct effects on pituitary thyrotropes and suppress blunt TRH-stimulated TSH levels [25]. This pathophysiological process may partly account for the dysfunction of the thyroid. As shown in Fig. 2, thyroid function slowly returned to normal after the surgeries were performed in 2016 and 2020, which was consistent with the decrease in serum cortisol levels. Therefore, we hypothesize that hypothyroidism is mainly due to hypercortisolism. It has been proven that hypercortisolism leads to dysfunction of the hypothalamic-pituitary-thyroid axis and can be reversible after surgery [14, 26, 27].

Surgery is the mainstay for local or locoregional P-
NETs. As a rule of thumb, a "watch-and-wait" approach is recommended as an option for P-NETs less than $2 \mathrm{~cm}$, especially for those with low grade and nonfunction. Formal oncologic surgery, such as Whipple resection or distal pancreatectomy/splenectomy, is recommended for P-NETs that are symptomatic, intermediate-to-high grade, or greater than $2 \mathrm{~cm}$ [28]. Resection of the primary lesion and reduction surgery of the metastatic tumor to minimize the secretion of hormones is consistent with domestic and foreign guidelines [28, 29]. In addition to resection of tumors or surgical cytoreduction, liver-directed therapies, including radiofrequency ablation and hepatic artery embolization, could also be applied to control liver metastasis [30]. Hepatic artery embolization likely benefits asymptomatic patients with 

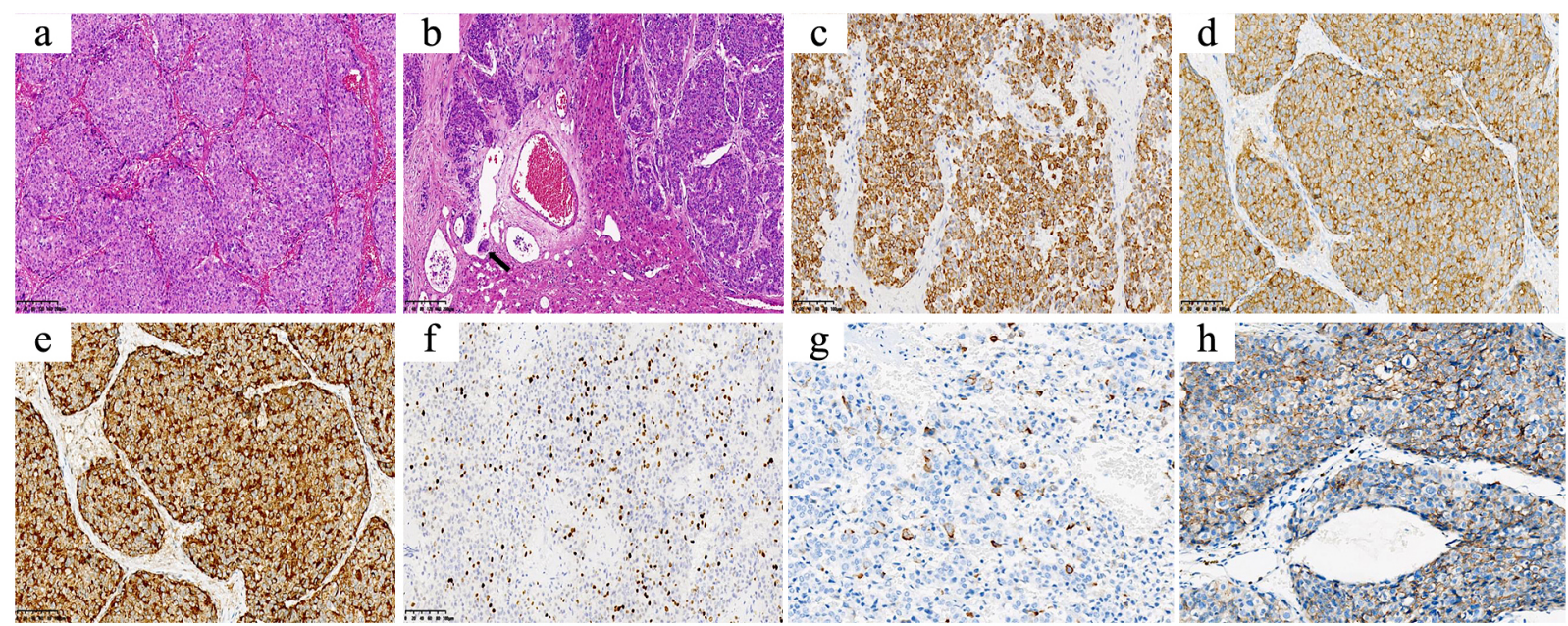

Fig. 4 Pathological examination and IHC of the hepatic tumor (January 2020)

a. Nodular growth of tumor; b. Tumor cells invade blood vessels (shown by the black arrow); c. Positive immunostaining for cytokeratin (CK7); d. Positive immunostaining for synaptophysin (Syn); e. Positive immunostaining for chromogranin (CgA); $\mathrm{f}$. The percentage of Ki67-positive cells was 15\%; g. Positive immunostaining for ACTH; h. Positive immunostaining for somatostatin receptor (SSTR) 2.
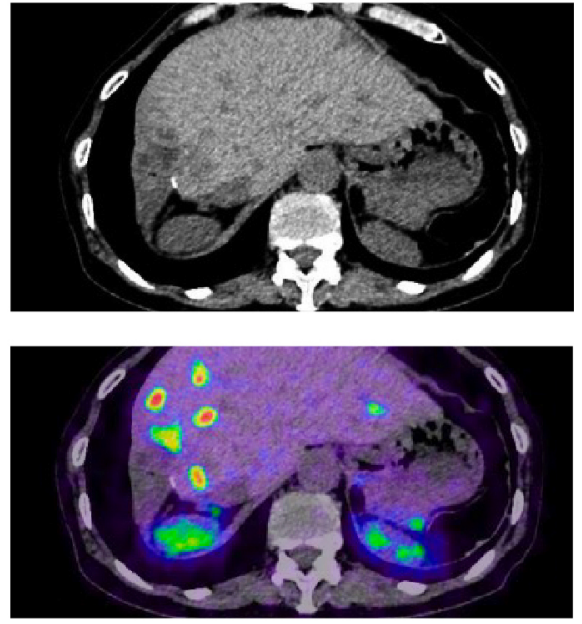
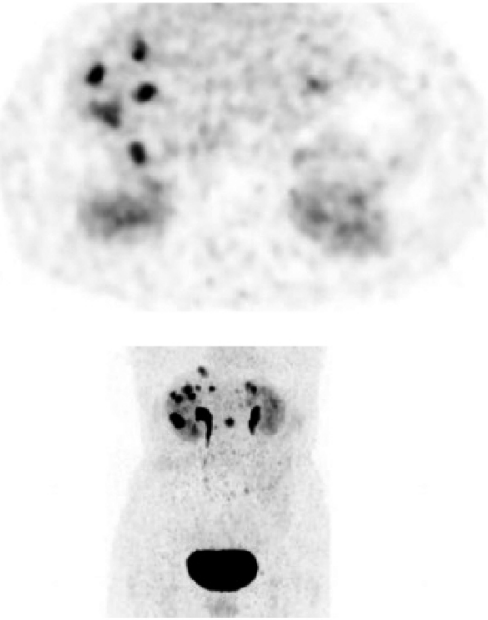

Fig. 5 PET-CT taken on April 2020 showed positive somatostatin receptor imaging in the liver

a large hepatic disease burden $(>25 \%)$ [31]. Liverdirected therapy also remains a key treatment modality for patients with hepatic dominant tumors [28].

In recent years, some new systemic treatments have been effective for metastatic P-NETs. Somatostatin ana$\operatorname{logs}$ (such as long octreotide, ranitide and parreotide), molecular targeted therapy (sunitinib and everolimus), interferon, chemotherapy (streptozocin +5 -FU and/or adriamycin regimen) and mifepristone [32-34] have been combined to relieve clinical symptoms and increase the survival rate of P-NETs. The therapeutic regimen of this patient is summarized in Fig. 6. Comprehensive treatment, including maintenance therapy with octreotide acetate microspheres, hepatic artery embolizations, and
CAPTEM regimen chemotherapy, was applied to control multiple liver metastases of P-NETs. Fortunately, the symptoms of EAS and the disease markers of the patient improved significantly after these therapies.

The prognosis of P-NET accompanied by EAS is poor. Tumors are usually aggressive and easily invade blood vessels and peripheral nerves. Metastases are often present when a tumor is first diagnosed, even prior to the clinical symptoms [35]. The median survival time of patients with G1 and G2 P-NETs with distant organ metastasis was 33 months, while that of patients with G3 stage disease was only 5 months [36]. The 5 -year and 10 -year survival rates were $35.0 \%$ and $16.2 \%$, respectively [37]. Specific subgroups of patients may have a 


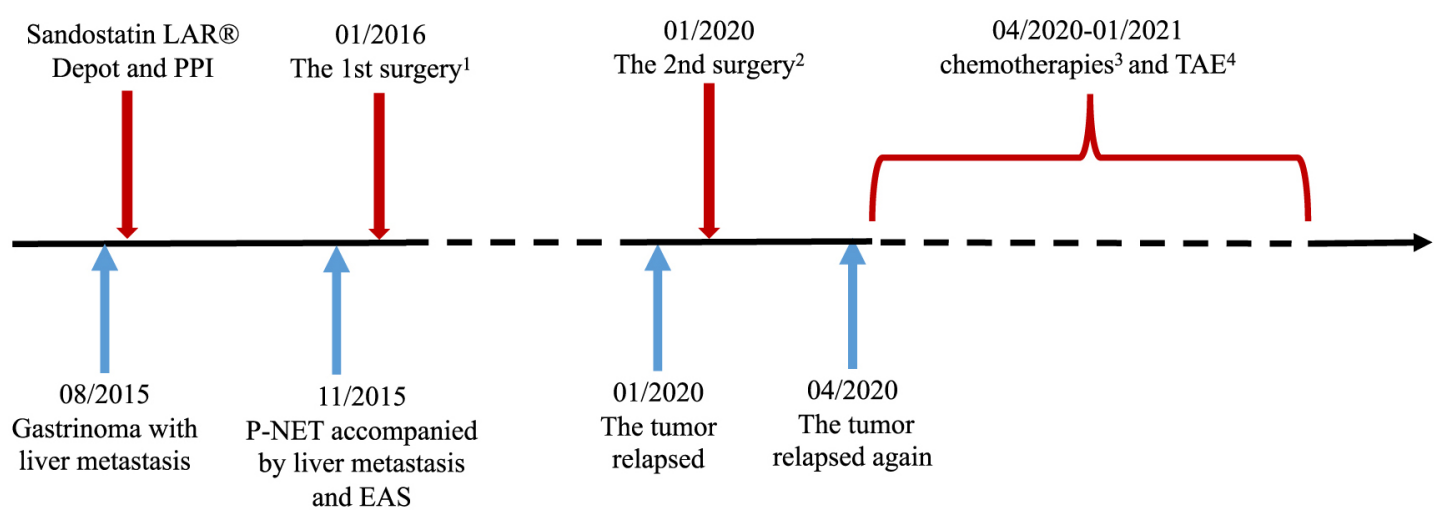

Fig. 6 Diagnosis and treatment of the patient

${ }^{1}$ Pancreatectomy, total splenectomy, partial liver tumor paresection and microwave coagulation cytoreduction

${ }^{2}$ Right hemihepatectomy, microwave coagulation of the left liver and cholecystectomy

${ }^{3}$ Twelve courses of capecitabine plus temozolomide (CAPTEM regimen)

${ }^{4}$ Three hepatic artery embolizations

higher risk of recurrence. Patients with a Ki-67 proliferative index of the tumor greater than 5\% may need more frequent surveillance, at least in the initial stage after surgery. The Commonwealth Neuroendocrine Tumor Research Collaborative/NANETS guidelines for surveillance suggest imaging every 6 to 12 months for 3 years and then every 1 to 2 years for at least 10 years in patients with $\mathrm{Ki}-67>5 \%$ and/or patients with positive lymph nodes.

In conclusion, P-NETs and metastatic lesions can simultaneously or successively secrete a variety of active hormones. Therefore, it calls for early identification of hormone secretion in primary care and nonendocrinologist specialists such as gastroenterologist or surgeons. IHC of multiple markers of biopsy specimens is also helpful for the diagnosis of P-NETs, especially in those secreting hormones without typical clinical symptoms. Tumor resection and surgical cytoreduction are still the basis of local tumor therapy. Systemic treatments, including SSAs, everolimus, interferons, chemotherapy, antiangiogenic agents, and liver-directed therapies, have expanded considerably to palliate hormonal symptoms and inhibit the tumor growth of P-NETs with metastasis. Frequent surveillance of hormones and images is recommended for metastatic P-NETs.

\section{Ethics Statement}

The authors obtained informed consent from the patient to share data and images.

\section{Author Contributions}

Mao Zheng, Li Chen and Xiaomin Nie contributed equally to this work.

\section{Acknowledgments}

We thank the patient for sharing the data with us.

This work was supported by the Research Fund of Anhui Medical University (NO. 2020xkj239) and the Fundamental Research Funds for the Central Universities (NO.WK9110000194.)

\section{Disclosure}

None of the authors have any potential conflicts of interest associated with this research.

\section{References}

1. Hubalewska-Dydejczyk A, Trofimiuk M, Sowa-Staszczak A, Gilis-Januszewska A, Baczynska E, et al. (2010) Neuroendocrine tumours of rare location. Endokrynol Pol 61: 322-327.

2. Xu JM, Tian XP, Wang ST (2013) Pancreatic neuroendocrine tumors. Chin J Endocr Surg 7: 333-335 (In Chinese).
3. Ito T, Sasano H, Tanaka M, Osamura RY, Sasaki I, et al. (2010) Epidemiological study of gastroenteropancreatic neuroendocrine tumors in Japan. $J$ Gastroenterol 45: 234243.

4. Mazzaferro V, Pulvirenti A, Coppa J (2007) Neuroendocrine tumors metastatic to the liver: how to select patients for liver transplantation? J Hepatol 47: 460-466. 
5. Patel FB, Khagi S, Daly KP, Lechan RM, Ummaritchot V, et al. (2013) Pancreatic neuroendocrine tumor with ectopic adrenocorticotropin production: a case report and review of literature. Anticancer Res 33: 4001-4005.

6. Zhao XD, Tian XD, Yang YM (2017) Diagnosis and surgical treatment of pancreatic neuroendocrine neoplasms. Chinese Journal of Hepatobiliary Surgery 23: 505-508 (In Chinese).

7. Maragliano R, Vanoli A, Albarello L, Milione M, Basturk $\mathrm{O}$, et al. (2015) ACTH-secreting pancreatic neoplasms associated with Cushing syndrome: clinicopathologic study of 11 cases and review of the literature. Am J Surg Pathol 39: 374-382.

8. Paragliola RM, Corsello A, Papi G, Pontecorvi A, Corsello SM (2021) Cushing's Syndrome Effects on the Thyroid. Int J Mol Sci 22: 3131.

9. Gu JQ, Sun WL, Zheng L, Hu HY, Cong HY (2017) Clinical analysis of thyroid function in patients with hypercortisolism. China Medicine 12: 243-245 (In Chinese).

10. Cintra A, Fuxe K, Wikström AC, Visser T, Gustafsson JA (1990). Evidence for thyrotropin-releasing hormone and glucocorticoid receptor-immunoreactive neurons in various preoptic and hypothalamic nuclei of the male rat. Brain Res 506: 139-144.

11. Kovacs K, Rotondo F, Stefaneanu L, Fereidooni F, Horvath E, et al. (2000) Glucocorticoid receptor expression in nontumorous human pituitaries and pituitary adenomas. Endocr Pathol 11: 267-275.

12. Kakucska I, Qi Y, Lechan RM (1995) Changes in adrenal status affect hypothalamic thyrotropin-releasing hormone gene expression in parallel with corticotropin-releasing hormone. Endocrinology 136: 2795-2802.

13. Alkemade A, Unmehopa UA, Wiersinga WM, Swaab DF, Fliers E (2005) Glucocorticoids decrease thyrotropinreleasing hormone messenger ribonucleic acid expression in the paraventricular nucleus of the human hypothalamus. J Clin Endocrinol Metab 90: 323-327.

14. Shekhar S, McGlotten R, Auh S, Rother LI, Nieman LK (2021) The hypothalamic-pituitary-thyroid axis in cushing syndrome before and after Curative Surgery. J Clin Endocrinol Metab 106: e1316-e1331.

15. Eigler T, Ben-Shlomo A (2014) Somatostatin system: molecular mechanisms regulating anterior pituitary hormones. J Mol Endocrinol 53: R1-R19.

16. Benker G, Raida M, Olbricht T, Wagner R, Reinhardt W, et al. (1990) TSH secretion in Cushing's syndrome: relation to glucocorticoid excess, diabetes, goitre, and the 'sick euthyroid syndrome'. Clin Endocrinol (Oxf) 33: $777-786$

17. Roelfsema F, Pereira AM, Biermasz NR, Frolich M, Keenan DM, et al. (2009) Diminished and irregular TSH secretion with delayed acrophase in patients with Cushing's syndrome. Eur J Endocrinol 161: 695-703.

18. Tamada D, Kitamura T, Onodera T, Hamasaki T, Otsuki $\mathrm{M}$, et al. (2015) Clinical significance of fluctuations in thyroid hormones after surgery for Cushing's syndrome. Endocr J 62: 805-810.
19. De Groot LJ (2006) Non-thyroidal illness syndrome is a manifestation of hypothalamic-pituitary dysfunction, and in view of current evidence, should be treated with appropriate replacement therapies. Crit Care Clin 22: 57-86.

20. Duick DS, Wahner HW (1979) Thyroid axis in patients with Cushing's syndrome. Arch Intern Med 139: 767-772.

21. Gamstedt A, Järnerot G, Kågedal B, Söderholm B (1979) Corticosteroids and thyroid function. Different effects on plasma volume, thyroid hormones and thyroid hormonebinding proteins after oral and intravenous administration. Acta Med Scand 205: 379-383.

22. Bános C, Takó J, Salamon F, Györgyi S, Czikkely R (1979) Effect of ACTH-stimulated glucocorticoid hypersecretion on the serum concentrations of thyroxinebinding globulin, thyroxine, triiodothyronine, reverse triiodothyronine and on the TSH-response to TRH. Acta Med Acad Sci Hung 36: 381-394.

23. Faglia G, Ambrosi B, Beck-Peleoz P, Giovanelli M (1973) Disorders of growth hormone and corticotropin regulation in patients with empty sella. J Neurosurg 38: 59-64.

24. Peng CX, Gao YM (2008) Clinical analysis of 44 cases of empty sella. Chinese Journal of endocrinology and metabolism 24: 486-488 (In Chinese).

25. Haugen BR (2009) Drugs that suppress TSH or cause central hypothyroidism. Best Pract Res Clin Endocrinol Metab 23: 793-800.

26. Xiang B, Tao R, Liu X, Zhu X, He M, et al. (2019) A study of thyroid functions in patients with Cushing's syndrome: a single-center experience. Endocr Connect 8: 1176-1185.

27. Cai R, Zhou W, Jiang L, Jiang Y, Su T, et al. (2020) Association between thyroid function and serum cortisol in cortisol-producing adenoma patients. Endocrine 69: 196-203.

28. Cives M, Strosberg JR (2018) Gastroenteropancreatic neuroendocrine tumors. CA Cancer J Clin 68: 471-487.

29. Pancreatic Surgery Group of Surgery Branch of Chin (2015) Guidelines for the diagnosis and treatment of Pancreatic Neuroendocrine Neoplasms (2014 edition). Chinese Journal of Hepatobiliary Surgery 30: 80-82 (In Chinese).

30. Strosberg JR, Cheema A, Kvols LK (2011) A review of systemic and liver-directed therapies for metastatic neuroendocrine tumors of the gastroenteropancreatic tract. Cancer Control 18: 127-137.

31. Mayo SC, Herman JM, Cosgrove D, Bhagat N, Kamel I, et al. (2013) Emerging approaches in the management of patients with neuroendocrine liver metastasis: role of liver-directed and systemic therapies. J Am Coll Surg 216: 123-134.

32. Wang XJ, Du TT, Zhang BP, Hu SH (2016) Ectopic ACTH syndrome caused by pancreatic neuroendocrine carcinoma-a case report. Acta Medicinae Universitatis Scientiae et Technologiae Huazhong 45: 238-240 (In Chinese)

33. Halfdanarson TR, Strosberg JR, Tang L, Bellizzi AM, Bergsland EK, et al. (2020) The North American 
Neuroendocrine Tumor Society consensus guidelines for surveillance and medical management of pancreatic neuroendocrine tumors. Pancreas 49: 863-881.

34. Pavel M, Öberg K, Falconi M, Krenning EP, Sundin A, et al. (2020) Gastroenteropancreatic neuroendocrine neoplasms: ESMO clinical practice guidelines for diagnosis, treatment, and follow-up. Ann Oncol 31: 844-860.

35. Bansal M, Agarwal A, Govindarajan R (2012) Ectopic adrenocorticotropic hormone syndrome due to a pancreatic neuroendocrine tumor. $J$ Gastrointest Cancer 43
Suppl 1: S231-S233.

36. Yao JC, Hassan M, Phan A, Dagohoy C, Leary C, et al. (2008) One hundred years after "carcinoid": epidemiology of and prognostic factors for neuroendocrine tumors in 35,825 cases in the United States. J Clin Oncol 26: 30633072 .

37. Nikfarjam M, Warshaw AL, Axelrod L, Deshpande V, Thayer SP, et al. (2008) Improved contemporary surgical management of insulinomas: a 25-year experience at the Massachusetts General Hospital. Ann Surg 247: 165-172. 